\title{
Agôn
}

Revue des arts de la scène

Critiques | Saison 2018-2019

\section{Sublimer la captivité}

Outside, mise en scène, scénographie et dramaturgie de Kirill Serebrennikov

\section{Caroline Châtelet}

\section{Q OpenEdition \\ Journals}

Édition électronique

URL : http://journals.openedition.org/agon/5758

DOI : $10.4000 /$ agon. 5758

ISSN : 1961-8581

\section{Éditeur}

Association Agôn

\section{Référence électronique}

Caroline Châtelet, « Sublimer la captivité », Agôn [En ligne], Critiques, mis en ligne le 31 juillet 2019 consulté le 02 août 2019. URL : http://journals.openedition.org/agon/5758 ; DOI : 10.4000/agon.5758

Ce document a été généré automatiquement le 2 août 2019

Association Agôn et les auteurs des articles 


\title{
Sublimer la captivité
}

\author{
Outside, mise en scène, scénographie et dramaturgie de Kirill \\ Serebrennikov
}

\section{Caroline Châtelet}

\section{RÉFÉRENCE}

Outside, de Kirill Serebrennikov, du 16 au 23 juillet 2019 à l'Autre scène du Grand Avignon - Védène, Festival IN.

$1 \mathrm{Au}$ sortir d'Outside, une amie me glissa, légèrement dubitative «Oui, enfin, ce spectacle ne serait pas reçu de la même manière s'il ne s'agissait pas de Kirill Serebrennikov ». Alors, certes. Le fait que l'artiste russe ait été assigné à résidence (sans téléphone ni internet) de l'été 2017 à avril 2019 pour une affaire présumée de détournement de fonds publics joue certainement dans la réception aimable de son travail. Peut-être, également, que ses démêlés avec la justice - dénoncés comme une cabale politique menée à l'encontre d'un artiste dont les engagements divers déplaisent au pouvoir en place (soutien au groupe Pussy Riot, lutte contre la limitation des droits des personnes LGBT, défense de la liberté d'expression, etc.) -, ajoutent à son aura. Le spectacle présenté cette année à Avignon n'oblitère d'ailleurs pas sa situation, puisque que les comédiens arborent lors du salut final un t-shirt « Free Kirill Serebrennikov ». Car si le tribunal qui le juge a mis fin en avril 2019 à l'assignation à résidence, Serebrennikov ne peut quitter Moscou, et l'équipe d' Outside est venue au festival sans son metteur en scène.

2 Néanmoins, ne s'agit-il pas toujours de cela? La personnalité et la position (symbolique, artistique, politique) occupée par un artiste n'influencent-elles pas de manière plus ou moins consciente, toute découverte d'une œuvre? À titre d'exemple, Architecture de Pascal Rambert créé cet été pour la Cour d'honneur du Palais des papes a été reçu à l'aune des précédents spectacles de son auteur et metteur en scène. C'est ainsi que nombre de critiques ont construit leur propos, inscrivant cet opus dans un parcours et déplorant son faible intérêt tout en vantant celui des précédents - dont Clôture de l'amour (le plus grand 
tube de Rambert, décliné comme un produit dans plusieurs langues et avec de multiples distributions).

D'autant que dans Outside, ce qui saisit ce n'est pas sa réception en regard de la situation vécue par son auteur/metteur en scène que la manière dont l'œuvre elle-même est irriguée par cette situation. À sa manière, le spectacle se donne comme une réponse aussi sublime que bouleversante à la captivité, la création sous surveillance. Ou comment l'imaginaire et l'art sont plus forts que l'enfermement - psychique, physique, politique. Mais reprenons. Lorsque les spectateurs prennent place dans la salle, le spectacle a déjà débuté. Sur un plateau à vue, occupé en son centre par un praticable, sorte de pièce de quelques mères carrés avec fenêtre, des colleurs d'affiche vaquent. D'abord deux, ils passent à trois, quatre, cinq, s'affairant pour afficher sur le mur du fond de scène une grande reproduction noir et blanc d'une photographie de l'artiste chinois Ren Hang. Au fur et à mesure du collage des bandes, l'image révèle une jeune chinoise allongée nue sur le parapet d'un bâtiment. Tandis que derrière elle l'on aperçoit le ciel et des immeubles typiques de l'urbanisme chinois, la femme regarde l'objectif. Un spectateur averti remarquera que le collage ne répond pas à la logique d'un tel travail, mais qu'il ménage la découverte progressive du corps. D'abord son visage, puis ses membres, l'ultime bande étant celle révélant son sexe. La manière dont les corps vont s'exposer durant tout le spectacle qui suit sera à cette image: rien de pornographique ni d'obscène, guère d'érotisme, plutôt une sensualité et une infinie douceur et délicatesse.

Une fois leur tâche accomplie, les colleurs d'affiche quittent la scène : comprenez que le décor, l'arrière-plan dans lequel ce spectacle trouve sa source étant posé - soit le travail du photographe Ren Hang - le théâtre en tant que tel peut débuter. Ce jeune photographe et poète chinois né en 1987 dans la province de Jilin au nord-est du pays, célébré à l'étranger (la Maison européenne de la photographie lui a consacré une exposition personnelle au printemps 2019), courtisé par les magazines et galeries en Occident, suivi par nombre de jeunes chinois sur les réseaux sociaux, devait en 2017 rencontrer Kirill Serebrennikov. Il se suicide peu de jours avant leur rendez-vous et le dialogue avorté se prolonge par la scène, la mise en scène s'attachant à donner à voir l'œuvre et la sensibilité de Ren Hang - par le regard et l'art de Serebrennikov.

5 Un homme incarnant Kirill Serebrennikov apparaît alors sur le praticable situé au centre de la scène, tandis que résonnent quelques notes sur un piano encore invisible. Assis sur le rebord de la fenêtre, en captivité dans ce petit espace qu'il ne quittera pas dans les premiers temps, il dialogue avec son ombre projetée au sol (interprétée par un comédien). Leur conversation est interrompue par une perquisition de la police et la scène balance alors entre réalisme et poésie. Si les costumes des policiers et de leurs supérieurs hiérarchiques sont réalistes, si la satire de la Russie fait mouche (l'embonpoint des policiers, le fait que l'une des membres de l'administration admoneste au téléphone son enfant durant la perquisition), la violence de la perquisition est métaphorisée. Les fouilles au corps et éventuels coups physiques deviennent des prouesses physiques drôlatiques. Il y a ici le même savoir-faire que celui déployé dans Leto, dernier film de Serebrennikov: la brutalité politique n'est pas reproduite, elle est déplacée, l'allégorie suscitant l'humour et l'onirisme. Ainsi procède la mise en scène qui va suivre, où les séquences vécues et racontées par Hang et Serebrennikov au plateau sont décalées par l'imaginaire.

6 Outside va, ainsi, déployer un dialogue impromptu, fondé sur les personnalités, les points communs des deux hommes (leurs rapports difficiles aux autorités de leur pays respectif, 
leur homosexualité, leurs doutes), les divergences, et explorant dans un geste amoureux et libre le travail du photographe. Les comédiens composent dans des tableaux vivants les images de Hang, tandis que résonnent ses textes poétiques. Dans ses prises de vue, l'artiste mettait en scène ses amis, sa mère puis, au fur et à mesure du temps, des personnes recrutées via les réseaux sociaux. Avec parfois tout un bestiaire (serpent, oiseaux empaillés, poules bien vivantes), ou seulement quelques fruits, du vernis à ongles rouges, des fleurs et des plantes, Hang explorait la nudité. Sans rien de pornographique, de voyeuriste ni d'obscène, et comme son personnage le confie dans le spectacle «je ne fais rien de dangereux, je photographie les choses dans leur état naturel ». Il se dégage, en effet, une pureté et une simplicité des corps exposés et de leurs postures ${ }^{1}$.

7 Dans un entremêlement d'images, de textes, de musiques - un orchestre d'abord caché puis intégré à la scène joue de bout en bout - les séquences se succèdent : la mère de Hang (qui refuse la mort de son fils), le lien fort à son pays, l'homosexualité, le goût pour la danse, la solitude. Tout se fait avec grâce, l'univers féérique savamment dessiné jouant de l'illusion et du simulacre. Il y a quelque chose de la fantasmagorie, que ce soit dans les couleurs acidulées, les paillettes, les chansons. À ce titre, la présence en continu de la musique allant de la pop au rock au classique ou à la techno participe de la puissance et de la cohérence de l'univers construit.

Cette pérégrination aux accents merveilleux ne se fait pas sans omettre le contexte politique. Mais là encore, Serebrennikov opère un détour par une parabole mâtinée de burlesque. Ainsi, un danseur russe au physique étrangement empâté, se présentant comme un sosie possible de Rudolf Noureev, traverse tout le spectacle. Ce n'est qu'à la fin qu'il raconte l'origine de son embonpoint : il a accepté de se faire pousser un «énorme cul » suite à l'ordre du directeur de ballet dont il fait partie. Cette soumission au pouvoir lui donne accès à une position reconnue, à la gloire, mais son art lui-même en est affecté.

D'aucuns pourront trouver certaines séquences de danse un brin naïves ou maladroites, le final - sorte de rituel de deuil - trop expressif ou illustratif. Peu importe. Parce qu'il se dégage d'Outside une énergie vitale et une liberté rares. D'ailleurs, pour qui aura vu l'un des précédents spectacles du metteur en scène russe (Serebrennikov a présenté en 2015 à Avignon Idiots ${ }^{2}$, d'après le film de Lars von Trier et en 2016 Les Âmes mortes de Nicolas Gogol), il est passionnant de voir comme chacun s'affirme comme une réponse scénique à un projet spécifique. [Digression/explication : là où tout le travail de Pascal Rambert, par exemple, répond à un même système d'écriture, de mise en scène, de direction d'acteur, les spectacles de Kirill Serebrennikov ouvrent à des esthétiques et des univers extrêmement différents, propre à chaque fois au propos abordé.] Là où Les Idiots se révélait très âpre et dur, Outside est irrigué par une singulière et sublime mélancolie. Tout en dialoguant avec Ren Hang et son œuvre, en lui rendant hommage, Kirill Serebrennikov et son équipe (tous des interprètes à la justesse impeccable), dessinent une œuvre d'une pure beauté. Soit une forme plastiquement fascinante, où les enjeux politiques et artistiques ne sont pas oblitérés, mais où la violence et les sentiments dépressifs sont conjurés par la sincérité et la croyance dans la puissance de l'art. 


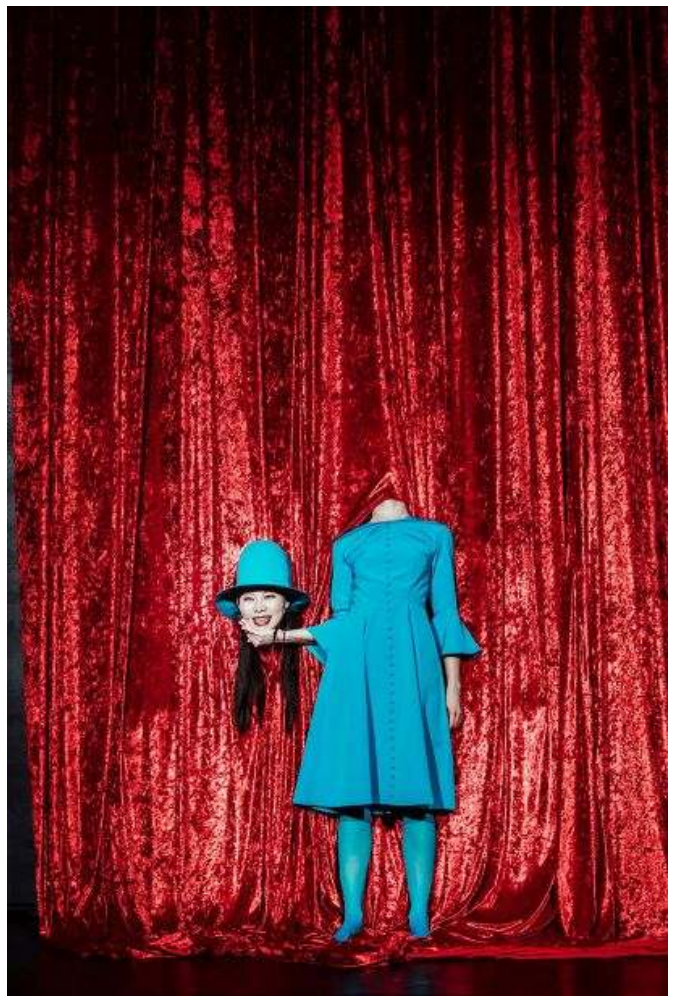

(c) Kirill Serebrennikov

\section{NOTES}

1. [Difficile de ne pas songer face à ce traitement du corps à la pièce chorégraphique de Gaëlle Bourges, À mon seul désir. Dans celle-ci, l'artiste explique que le nu n'existe pas dans la nature. De la même manière, le nu n'existe pas comme objet impudique chez Ren Hang, il est une chose naturelle.]

2. "A idiots, idiots et demi ", critique des Idiots, publié en juillet 2015: https:// journals.openedition.org/agon/3222

INDEX

Mots-clés : Kirill Serebrennikov, Russie, Ren Hang, festival d'Avignon 